\title{
Good Intuition or Fear and Uncertainty: The Effects of Bias on Information Systems Selection Decisions
}

\author{
Kieren Jamieson and Paul Hyland \\ Central Queensland University, Rockhampton, Australia
}

k.kamieson@cqu.edu.au p.hyland@cqu.edu.au

\begin{abstract}
IS selection decisions are traditionally viewed through a techno-rationalist lens; however, it is clear that numerous biases affect the decision makers. In this paper, we have categorised common types of biases into four groups. Firstly, information biases distort information and how it is weighted. Secondly, cognitive biases are "games" decision makers play to simplify information processing. Thirdly, risk biases distort the way information is used in order to minimise risk. Finally, uncertainty biases act to reduce the uncertainty surrounding decisions. In this paper, we have developed a framework for conceptualising how these biases interact and affect decisions.

After introducing the framework, we use it to examine specific Information Systems acquisition decisions in two organisations. The first organisation is a not-for-profit Australian health and aged care group that purchased a Patient Management System. The second organisation is an Australian higher education institution that purchased an Enterprise Resource Planning system. The paper concludes that the framework is useful; however context has an important role in determining the effects of bias on decision outcomes.
\end{abstract}

Keywords: Bias, Uncertainty; ERP, Decision Making

\section{Introduction}

Decisions relating to the selection of Information Systems (IS) are becoming increasingly complex and at the same time critical to many organisations. Decisions are the outcomes from the process of assessing and evaluating factors and decision making is the act of choosing among alternatives (O'Reilly, 1990). The informing systems that affect decision making are influenced by a number of contextual organisational factors including cost, time and resource availability (Simons \& Thompson, 1998). Though a wide body of literature exists on decision making processes and models (see for example March \& Simon, 1958; Mintzberg, Waters, Pettigrew \& Butler, 1990; Simon, 1997), little research has been conducted about the informing systems and in-

Material published as part of this journal, either on-line or in print, is copyrighted by the Informing Science Institute. Permission to make digital or paper copy of part or all of these works for personal or classroom use is granted without fee provided that the copies are not made or distributed for profit or commercial advantage AND that copies 1) bear this notice in full and 2) give the full citation on the first page. It is permissible to abstract these works so long as credit is given. To copy in all other cases or to republish or to post on a server or to redistribute to lists requires specific permission and payment of a fee. Contact Publisher@InformingScience.org to request redistribution permission. fluences affecting senior managers making decisions relating to IS: one such group of influences is bias. Biases distort and filter the information available to decision makers and though there is considerable literature from the management and psychology disciplines describing the effects of bias on decision making, the nexus between such biases and IS decision making outcomes is poorly documented and understood. 
The limited amount of research into IS decision making has revealed that IS decision making is rarely logical or rational (Bannister \& Remenyi, 1999). This is contrary to conventional thinking that the decision to purchase or implement an Information System follows standard large capital acquisition practices and is the result of a formal evaluation with logical and rational justifications (Ballantine \& Stray, 1999; Doherty \& King, 2001; Irani \& Love, 2001; Lin \& Pervan, 2001; Verville \& Halingten, 2002).

The lack of rationality in many IS decision making processes can often be traced back to contextual influences, many of which are intangible biases (Jamieson \& Hyland, 2004). This study seeks to highlight and describe the effects of these biases on IS decisions outcomes by examining their role in two large IS projects. This research aims to establish ways of mitigating negative effects of bias, while modelling its role in complex IS evaluations.

The paper begins with an overview of this study where a model of the interaction between information, bias and decision making is described. The theoretical background to the research is detailed by describing four groups of biases that affect informing systems. The case study methodology and analysis techniques are then outlined. The paper then presents findings of empirical research with a focus on the bias groupings identified from the literature. The paper concludes with a discussion and analysis of the findings and presents a revised version of the theoretical model.

\section{Theoretical Background}

As Simon (1987) noted, intuition does not operate independently of logical analysis as the two elements are complementary in a decision making processes. While Simon understood that elements such as stress play a part in the rationality of decision making processes, he also noted that there are a number of cognitive and environmental biases that also affect decisions. These biases, in combination with other contextual factors, can radically affect the way information is gathered and processed within the informing system.

Four groupings of biases that affect decision making are prevalent in the literature. These are information biases, cognitive biases, risk biases and uncertainty biases. For the purposes of this study, their effects on informing systems, information and the way information is processed has been developed into a model as depicted in Figure 1.

In this model, bias affects decision making in two ways. Firstly, information flows into a decision making process and in doing so may pass through one or more bias lenses of filters. A bias lens is a conceptual view through which decision-makers process information. The lens has the potential to screen, alter or intensify the information that enters it. An example of this is an information bias that distorts the perceived benefits of a decision option. Secondly, biases can influence how the decision occurs by shaping the process. An example of this is an uncertainty bias that restricts or stops the search for informing factors.

Not every bias group will be present in all organisations and decisions. However, from the literature, it is clear they have a role in decision making. In this section, each of the bias groups and their effects are described in order to provide the theoretical background to the research and for later justification of the findings and development of the model. 


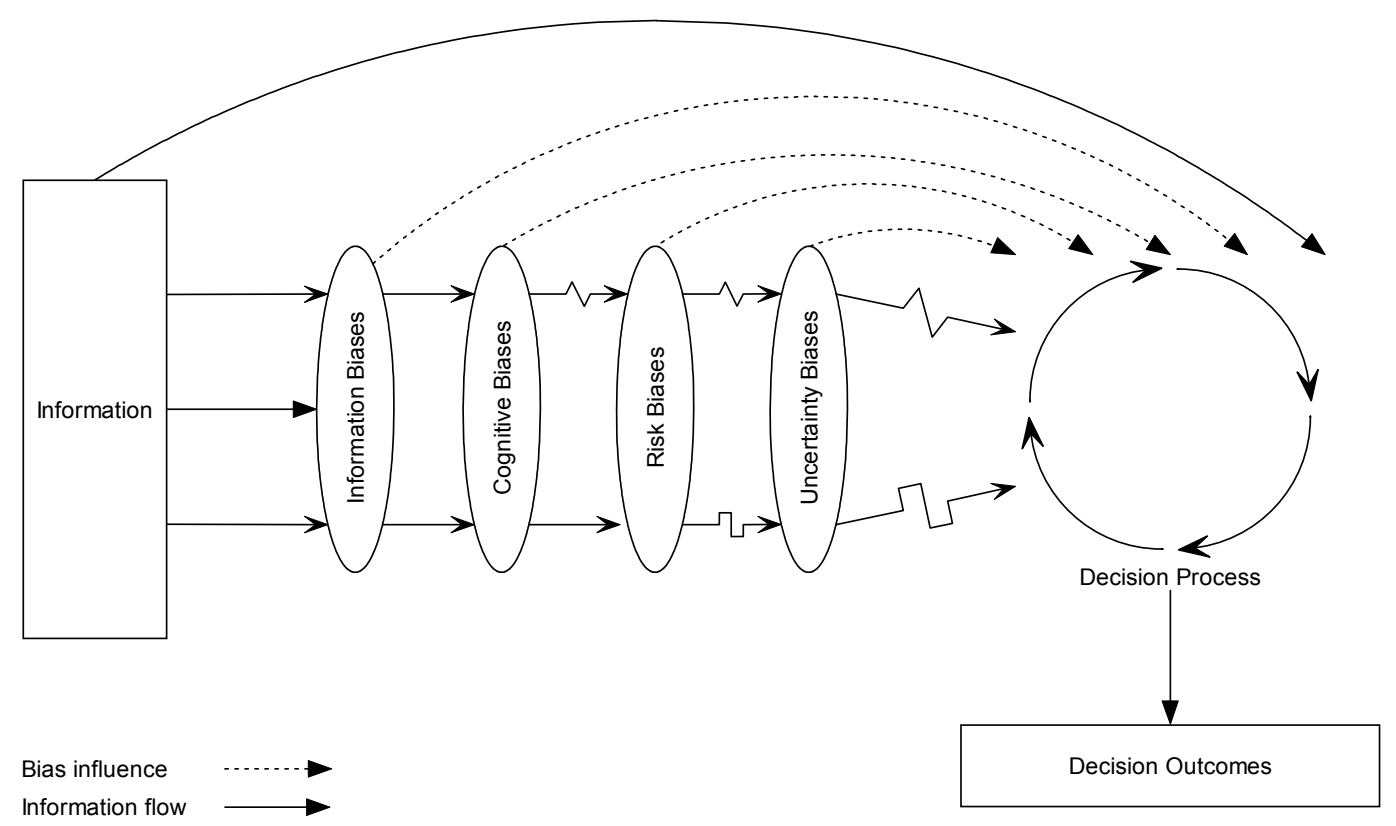

Figure 1 - Bias in an informing system

\section{Information Biases}

Information biases are the first group filters and influences that affect decisions. This is because, when choosing amongst alternatives, decision makers often unconsciously distort information. This is known as desirability bias, optimism, outcome bias, value bias and wishful thinking (Russo, Meloy, \& Medvec, 1998). In their study of pre-decisional information distortion, Russo et al. found that the formation of preferences occur without instruction and this can lead subsequent pre-decisional distortion of product information. They also found that pre-decisional distortion is proportional to prior confidence in the leading alternative. This means that even when decision makers are not asked to choose an alternative based on information presented to them, they still intuitively form a preference. This preference then biases or distorts their decision making process. Russo et al. also found that even when presented with clear, factual, non-subjective decisionrelevant diagnostic information that presented contrasting information between alternatives, people still exercised individual pre-decisional distortion. They note that pre-decisional distortion presents a genuine risk to choice accuracy and provided a reason for this bias:

The desire to reduce effort might lead to pre-decisional information distortion, as follows: Distortion builds confidence in the leading brand, which enables a sufficient level of confidence to justify stopping the search for product information sooner than it would have been without distortion. Earlier stopping saves the effort of additional information search (Russo et al., 1998, p. 448)

This behaviour reinforces the satisficing decision making in Simon's (1967) rational model and his view of administrative behaviour and the administrative man (Simon, 1997). This means that while information biases can distort information, they can also alter the way in which the search for information occurs. 


\section{Cognitive Biases}

In order to simplify decision making, a second group of biases, Cognitive biases, are applied to decision making. Duhaime and Schwenk (1985) argued that the amount of information available to decision makers often exceeds the decision makers' processing limits. Since decision makers are often unable to cope with all the information relevant to a decision, they simplify the decision making process by applying cognitive filters or biases. Brindle (1999) refers to these biases as cognitive games. The four main biases that Duhaime and Schwenk (1985) have identified are reasoning by analogy, illusion of control, escalating commitment and single outcome calculation.

\section{Analogy}

Reasoning by analogy is the application of analogies from simple situations to complex strategic problems. Duhaime and Schwenk note that this scaling of analogies from simple to complex problems can lead to an over simplification of the information to be considered in making the decision. Brindle (1999) calls this bias the misuse of analogy game and described it as the process of comparing and referencing other past decisions to the current decision under consideration. This analogy provokes a subtle emotional bias that causes the decision maker to either focus on or ignore certain information, depending if the information was relevant to the decision that it is being compared to. It will also lead to elimination of decision alternatives if they were similar to failed selected alternatives in previous decisions. Finally, misuse of analogy can be used to build support for a decision alternative. This occurs when an alternative is the same or similar to the alternative in the previous decisions.

\section{Illusion of control}

The illusion of control is where decision makers overestimate the extent to which the outcomes of a decision are under their personal control and their ability to correct or fix problems should they arise as a result of a decision. There is also an overestimation of the personal ability of the decision maker to actually make the decision. This form of bias tends to occur in individuals who have experienced prior success in complex decision making. Decision makers affected by this bias tend to focus on the parts of the decision they can control and not think about the factors that exhibit uncertainty. They also overestimate their own capabilities in order to reassure themselves in the face of uncertainty.

\section{Escalating commitment}

Escalating commitment is the tendency for a decision maker to maintain and increase support for a decision, even if the decision appears to be having negative consequences. An example of this occurs when a decision is made to acquire a product. Following the decision, there is a significant feeling of personal responsibility by the decision maker to remain with the product even if it is not performing, thus the bias in this example would deter divestment. This is a potentially harmful bias as there is evidence of its negative effects, particularly on Information Systems projects, in the literature (Mahaney \& Lederer, 1999; Smith \& Keil, 2003).

\section{Single outcome calculation}

Single outcome calculation is the restriction of decision alternatives to the most promising ones as determined by shared beliefs within the organisation at the time of the decision. This provides a rapid convergence of options but restricts creative alternatives. This restriction of alternatives can be as severe as a single option without any search for alternatives. This is common in the case of divestment where the organisation reaches the collective belief that a failing capital investment decision must be reversed. This type of bias reduces stress in the decision making process. 
Other cognitive biases or games include the framing game, the criteria selection game and the rationality game (Brindle, 1999). The framing game is concerned with the way a problem is defined and constrained. If a problem's dimensions are reshaped, this will affect the information sought and decision alternatives. Once framing occurs, there is a commitment to the way in which the decision is being made. This can be thought of as a combination of Bainbridge's (2002) overconfidence bias and Duhaime and Schwenk's (1985) escalating commitment. Decision makers become attached to their understanding of the problem and the decision alternatives. They become increasingly less predisposed to admit new information and alternatives into the decision making process. Sometimes framing is simply a case of not looking at the real problem (Brindle, 1999). Brindle (1999, p. 609) notes "sometimes, decision makers are not aware of their real agendas, but present the problem, often with perfect integrity, as the way they 'see' it".

The criteria selection game is described as the bias decision makers have towards measurable, quantitative data as opposed to less measurable qualitative data (Brindle, 1999). Decision makers like simplified, easy to understand data, even though this may omit details that are necessary in the decision process. Maritan (2001) supports this view with her findings that quantitative data is more heavily relied on for justifying capital investment projects. Visual data, such as graphs and charts, will be more important to decision makers than qualitative arguments (Brindle, 1999). As discussed later, this "game" is the result of uncertainty and the need to reduce information load.

Brindle (1999, p. 611) calls the rationality game as "the most insidious game". This bias occurs when decision makers produce rational arguments to constrain the information search or list of alternatives. The underlying reasons for these rational constraints are not examined and are not transparent. These restrictions constrain the decision maker and the ultimate quality of the decision. The key point is that the underlying reasons are not challenged. There is a degree of crossover between this game and Duhaime and Schwenk's (1985) concept of single outcome calculation in that organisational beliefs quickly constrain the alternative list.

It is important to understand that these cognitive games are not deliberate attempts by decision makers to distort information or the decision making process. The application of these biases is a reaction to the complexity of decisions and the need to simplify the management of information. It should also be evident that bias groups do not act in isolation of one another.

\section{Risk Biases}

Risk biases form the third group of filters and influences that act on the decision process. These biases mainly act on decision alternatives and minimising risk during the decision process. The perception of risk can bias decision outcomes because many of the information and cognitive biases that are used by decision makers are also used to mitigate risk. Conventional decision making theory suggests that choice is a combination of risk and expected gain. Decision makers who are risk averse choose alternatives that may have lower potential gains, with smaller variations of outcomes and with relatively low risk. Decision makers who are risk seeking choose alternatives that potentially provide higher gains, though with greater variation in outcomes with higher risks (March \& Shapira, 1987). However, based on an unpublished work of Shapira's (Shapira, 1986, as cited in March \& Shapira, 1987), March and Shapira provide a number of insights into managerial perceptions of risk that showed variations from conventional decision theory.

The first area concerned the definition of risk and concerning this, they had three findings. Firstly, they have found that in weighing up decision alternatives, managers do not treat uncertainty about positive outcomes as risk. Risk is only associated with potential negative decision outcomes. Secondly, managers view risk in terms of magnitude, not probability. Managers view risk in terms of how much negative impact a decision alternative could cause, not the combination of probability and the impact of the decision. Managers look for worst outcomes or maximum losses which in 
themselves do not indicate risk. Thirdly, while managers discuss and seek precision in measuring risk in quantifiable terms, most managers are not interested in reducing risk to a single quantifiable figure or formula. This is perhaps not so surprising given the difficulty in establishing such metrics in the first place.

The second area concerns attitudes towards risk. March and Shapira (1987) have found that the perception of risk and risk taking varies depending on the position in the organisation. For example, senior managers see risk taking as important and were more likely to take risks. However, March and Shapira have found that practically, managers were individually risk adverse within organisational structures, encouraged group decision making and did not encourage risk taking. This contrasted with their findings relating to managers' beliefs about their individual risk taking. Managers believed that they were more judicious and less risk averse than their colleagues, while also believing that they were greater risk takers than they actually were. Managers believed that risk taking was essential to their roles and acknowledged the emotional pleasures that risk taking provided. However, taking risks also depends on the context of the decision. If a decision maker is operating above the expected performance target, they are less likely to take risks. If the decision maker is operating below target, they are more likely to take risks.

The third area concerned dealing with risk. Managers were reported by March and Shapira (1987) as believing that risk was a manageable issue and made the clear distinction between risk and gambling. While gambling involved inherently uncontrollable risk, risk taking involved the reduction of uncertainty and risk modification. Managers would seek to reduce the probability of negative alternative consequences before making the decision. However, managers often do this by reassessing or modify the risk associated with a given alternative, either by recalculating the risk or including controlling strategies. This behaviour is an application of information and cognitive bias. It is clear from these findings that risk has some influence on decision outcomes and processes. However, in order to mitigate risk, decision makers seek to reduce uncertainty. This relationship with uncertainty biases is explored in the next section.

\section{Uncertainty Biases}

The final grouping of biases is Uncertainty biases. These filter and influences act on information and process in order to reduce uncertainty in the decision maker. The level of uncertainty surrounding a decision creates a bias that alters the way in which information is gathered and the decision is made. In order to reduce uncertainty, decision maker often use cognitive games, or biases. Uncertainty is the perceived gap between the information available and the information a decision maker wants to have (Buchanan \& Kock, 2000). It is the difference between the knowledge required to make a decision and the knowledge a decision maker has at that time and it is often inversely proportional to the decision-maker's level of understanding of the problem (Falzon, Zhang, \& Davies, 2000).

Uncertainty influences both the decision maker and the outcome of the decision and occurs when the decision maker is unable to assign definite probabilities to the consequences of a decision (March \& Simon, 1958). In order to reduce uncertainty, decision makers often attempt to acquire more information. Daft and Lengel (1990) indicate that as part of this behaviour, decision makers often gather and rely on more information from external sources, especially if there are limited internal sources available. However, as discussed later, some research suggests that the acquisition of additional information is not necessarily informing better decisions or reducing uncertainty (Buchanan \& Kock, 2000; Chan, 2002; Grise \& Gallupe, 2000; Iselin, 1993) and there is considerable evidence to suggest that providing additional information can increase uncertainty levels (Bartlet \& Green 1966, Dudycha \& Naylor 1966, Khon 1974, and Woodruff 1972, as cited in Jacoby, 1977). 


\section{Information}

The measurement of the information used in decision making is described as Information load. Information load is "the variety of stimuli (it consists of all data and information available to the decision maker) to which the receiver must attend" (McCormick, 1970 p. 114). It consists of external stimuli, dimensions of information, diversity of information and alternatives (Grise \& Gallupe, 2000). Iselin (1993) separates the concepts of information and data load by defining data load as the number of cues or pieces of data that were not relevant to the decision and information load as the number that were relevant to the decision. This meant that, of the data relevant to the decision, only a given proportion of it could be used as information directly informing the decisions, while the remainder, the data load, was simply discarded. It was found that increasing the data load resulted in poorer decision quality (Iselin, 1993).

Part of the problem associated with the way decision makers process information is the way in which data is presented. Decision makers may be more effective when they are presented with data in a form that has a greater cognitive fit with their decision making processes (Mintzberg, 1972; Umanath \& Vessey, 1994). If data can be manipulated and presented in a more effective way, this may reduce information load, for example, presenting data graphically rather than in tabulated form (Umanath \& Vessey, 1994). There is evidence to suggest that data manipulation leads to more accurate and quicker decisions (Vessey, 1994) although more recent studies have suggested that simply converting data into graphs does not necessarily reduce information load (Chan, 2002). Even so, a cognitive game is played to reduce uncertainty by selecting information that aligns with the decision making process.

\section{Information overload}

Although uncertainty provokes decision makers to seek more information, increasing information may not decrease uncertainty. As Schroder, Driver and Streufert (1967) argue, there is a limit to the amount of information that can be integrated into the decision making process. They maintain that the information absorption peaked, and then declined, as environmental complexity, or the amount of information available to the decision maker, increased. This behaviour is described as Information overload and results from "the finite limits of the ability of human beings to assimilate and process information during any given unit of time" (Jacoby, 1977, p. 569). It is a direct result of too much information for the available information processing capacity (Schick, Gordon \& Harka, 1990, as cited in Chan, 2002). Information overload has been identified as a problem in the management of information systems as it impedes the organisation and analysis of ideas and alternatives (Grise \& Gallupe, 2000). As information load increases, so does the instance of information overload (Grise \& Gallupe, 2000). This problem has been referred to as "Information Fatigue Syndrome" (Buchanan \& Kock, 2000). If the increase in information creates an information overload, then decision makers have greater uncertainty as they are not only unsure of the decision outcomes but they are also unsure of which information is most relevant to the decision making process.

The result of this uncertainty is a vicious circle because as information load increases, the proportion of information sought decreases while the number of alternative decision outcomes sought increases (Swain \& Haka, 2000; Umanath \& Vessey, 1994). This means that as a decision maker is faced with an increasing amount of information relating to a decision, they choose to seek less of it while also searching for more possible decision outcomes or options. This means that the more complex a decision is, the less informed, systematic and thorough the decision making process will be. This has an adverse effect on decision quality (Chan, 2002) and as Chan (2002, p. 3 ) notes, "providing more information than they [decision makers] can accommodate will reduce their problem solving effectiveness and lead to poor decisions". 
The overall effect of the uncertainty biases is one of recursive cognitive games until the decision makers perceive that their levels of uncertainty are lowered to acceptable limits. These games often rely on either selecting data that aligns with their decision making style, or by applying complex uncertainty reduction thought processes similar to the heuristic-systematic decision making style (Eagly \& Chaiken, 1993). The net effect is that the perception of uncertainty can lead to sub-optimal decision outcomes.

\section{Methodology}

Qualitative data for the study was gathered from ten interviews with key stakeholders in two organisations that had recently implemented large information systems. One organisation was from the private sector (Organisation A) and one was a government funded education institution (Organisation B).

Organisation A is a not-for-profit Australian health and aged care group based on the religious philosophy of care and charity. The group operates over a number of facilities across regional centres, consists of three acute care, one aged care and various support facilities such as food preparation, laundry and central administration. The organisation was originally established in the early part of the $20^{\text {th }}$ century by a religious order and now has more than 1100 employees. Organisation B is an Australian higher education institution spread over multiple sites throughout Australia. In addition, it maintains commercial operations in a number of Australian states in capital cities as well as commercial agreements with offshore delivery partners.

The two organisations examined in the case studies were selected because of a number or core commonalities in their needs and drivers for the implementation of the IS. Both projects had similar complexity and similar organisational risk associated with a failed selection and implementation process. However, the organisations differed in many other contextual ways which provided an insight into varied culture, organisational history, decision making style and politics. Interviewees were selected based on their involvement with the selection and implementation processes. Senior managers, members of decision making bodies and people who informed the decision process were interviewed from multiple levels and functional areas. This selection of interviewees gave a broad range of views and a richness and depth to the data gathering.

Additional data was obtained from organisational documentation and media reports. Interviews and documentation were synthesised into case narratives and a text based analysis was performed to obtain key themes and outcomes. Detailed information on the methodology is beyond the scope of this paper, however, the interested reader should note that the approach taken follows Yin's (1994) case study methodology. The methodology was selected because of the depth and richness of data that can be gathered from qualitative case studies. The analysis was guided by Klein \& Myers' (1999) seven principles of interpretive research and is based on the hermeneutic technique used successfully by Myers (1994a, 1994b) in his examination of IS project failures in New Zealand. As such, findings are presented as a part of an overall case narrative with contextual information provided to explain or describe the effects of the behaviours and biases. It should be noted that a statistical treatment of the data is inappropriate because of the small sample size and the aim of the research in describing the effects of bias in IS projects.

\section{Findings}

The study found considerable empirical evidence of bias in decision making in both organisations with both positive and negative impacts on decision outcomes. This section details the findings of the research by contrasting the data from the two cases. Each project is described and then evidence of the effects of each of the four bias groups is detailed. A summary of the effects of the bias on each organisation completes the analysis for each case. 


\section{Organisation A}

Organisation A required a replacement for its electronic patient management system. The legacy software, a Patient Management System (PMS) was introduced in 1984 and used terminal based technology. This system was maintained and upgraded in response to changes in the health care industry and government regulations. As well as patient management functionality, the product contained an integrated financials module used for billing, accounts payable, accounts receivable, general ledger and assets. During the life of the legacy software, it went through four software vendors. The second last vendor signalled their intent to "de-support" the product and gave users four months notice to move to another product, however, they withdrew the deadline due to pressure from large users and continued to support the product. Finally, Vendor A1, the final owner of the legacy software, announced the date at which it would no longer be supported and offered a replacement system, Product A1. As interviewees noted, the "old system worked fine" and it "was a very stable system", just that it was no longer supported. Therefore, a decision was made to explore alternative systems and make an implementation decision. Ultimately, the vendor of the existing legacy system was selected to provide the new patient management solution. The organisation implemented the system and there was a high level of satisfaction in the outcomes.

\section{Evidence of information bias}

Evidence of pre-decisional bias was provided by the Group Executive Officer (Business). He justified the selection of the successful vendor's product by stating it was strategically aligned with the needs of the organisation and that there were good support relationships in place. However, he then went on to make the comment that the "incumbent was first cab off the rank". This theme was prevalent throughout the interviewees in the organisation who viewed the existing vendor's product as the one that was always going to be the likely choice. Interviewees trusted the information from the vendor, and with this view in mind, benchmarked other vendor's products against the incumbent's. The Chief Financial Officer (CFO) remarked on the bias towards the incumbent vendor stating that there was always a "lean towards them" and "at the end of the day if you have two products that stack up fairly well and you know that their old support has been good, then you are going to go with the one that you know".

\section{Evidence of cognitive bias}

There was an indication that single outcome calculation was occurred during the decision making, however, the effects were mainly positive. The organisation has a cohesive culture and strong shared belief system. Much of the decision making and evaluation was about guiding the organisation through the process to an inevitable single conclusion. The indication from the interviews was that the evaluation process was not so much about evaluating products as effecting organisational change and user acceptance. The Group EO (Business) stated that "the culture of our organisation is consultative so this process was consistent with this". As the Information Officer stated, "It wasn't a hard decision to make in the end as there were not a lot of choices. It was quite obvious at the end".

Another example of this bias, in combination with the rationality game, came about when it became clear that the preferred vendor was not able to provide an integrated financials package. However, the preferred vendor did offer a separate financial system that integrated closely with their product, although it was based on UK market requirements. This product was not regarded as entirely suitable: "when we did the evaluation, we would say that the financial system didn't have the same robustness of the Patient Management System but we went for integration over that", noted the Group EO. The vendor later withdrew the product, offering an alternative from another vendor with customised interfaces to the Patient Management System and this was ac- 
cepted by the organisation. When the CFO was asked if other finance system options were considered, she replied:

No...I guess we were a bit pre-locked into the financials we went to, as we'd started on the patient management system, they had made a decision on the system we were going for, and that group had a particular financials package ... it wasn't until we'd gotten part of the way down the track that they pulled out and offered an alternative financials package ... so we really just took that and went through an implementation study with them.

However, the CFO had some reservations:

I'd like to have had the time to investigate more systems because we didn't know if there was anyone else out there that could have supported or interfaced with us as effectively as we believed [the selected financials product] would. We now know of at least one other player out there who could have done.

The CFO also stated that, "if you were doing it again, you'd obviously evaluate more than one system". This evidence indicates a form of escalating commitment on the part of Organisation A. They were locked into one vendor and had already decided to go with whatever finance solution the vendor provided. This created a form of single-outcome mindset, leading to the null evaluation and choice of the default product.

\section{Evidence of risk bias}

Organisation A's management could easily be classified as risk averse, having a conservative approach to organisational management and strategic direction. However, at the time of the PMS selection decision, there were additional pressures. The hardware running the legacy system was out of warranty and the Information Systems Officer noted that "It was time to make a change anyway". The risk adverse nature of the organisation and its decision makers made them give more weight to such factors as the long term relationship with the vendor and how well they would be supported. The Project Manager noted that:

A big part of it initially was the relationship ... one of the biggest things ... you need to get a feel whether you're going to be there in ten years time with these guys ... certainly cost is a major factor but its not the highest factor ... the fact that they were going to be a good partner for ten years was probably one of the highest thing ...you get a gut-feeling of how they behave and their history at other sites.

The Group CEO stated that "You get a sense... a gut instinct ... about how supportive these people are going to be". To minimise the risk to the organisation, the project manager also negotiated a contract that included penalty clauses for failure to deliver support.

\section{Evidence of uncertainty bias}

Organisation A approached the decision surrounding the PMS selection with a low degree of uncertainty. This meant that the effects of uncertainty biases were minimised, leading to more focused information gathering. While it could be argued that this artificially narrowed the search options, the decision makers were already familiar with many of the vendors and products on the market. As an example, the Group EO stated that:

We were not unfamiliar with competitor because part of the evaluation was the representative from the opposition sites come to the software evaluations of both products ... their scores counted to the ultimate outcome and we had and we did a review in the early $90 \mathrm{~s}$ of [on alternative product] and the product we saw in 2000 was no different than the one we saw several years ago except that it had a web browser over it. We were not con- 
vinced that a legacy product with a web browser on it would give us the advantages and the information together as going with [the selected product].

Perhaps this low level of uncertainty was because of a perceived lack of alternatives. The Group EO noted that:

Look, there might have been three or four systems we started with and each time we narrowed it down, we dropped one off the list because we thought it would not deliver what we wanted. We didn't want to go from one system to another and not gain anything ... not have spent all that time and money and not come out the other side not being able to access the information we needed.

The low level of uncertainty meant that the decision was rational and transparent, reducing the effects of other biases.

\section{Effects of bias in Organisation A}

Organisation A engaged in a structured, formal, decision making process. However, the actual motivation for this process was just as much about effecting organisational change and acceptance as it was about choosing between products. From the outset, evidence suggests that there was bias towards the incumbent vendor's product offering. This was explained using words like "confidence" and "comfort" in relation to how the organisation felt towards the vendor. This lowered the level of perceived risk and uncertainty. Although there was some evidence of single outcome bias, the decision making generally appeared rational and was both technically an organisationally justified.

One negative effect came from the single outcome/rationality bias on the selection of the financials system. This decision seems to have come from a null search without considering other viable options. This may account for the mild level of dissatisfaction with the decision and decision making displayed by the CFO.

Overall, the pre-decisional bias exhibited was justified by the relationship the organisation had with the vendor. The positive outcomes from the transparent and rigorous evaluation process provided the organisation with confidence in the decision and the outcomes. It was the transparency and rigour of the evaluation process that limited the effects of other bias groups.

\section{Organisation B}

In 1993, the Organisation B's IS division became involved in an Australia-wide project for developing a core specification for a higher education administrative systems. The Core Australian Specification for the Management of Administrative Computing (CASMAC) was initiated in 1991 with Australian higher education institutions following one of three different software implementation paths. These consortiums were advantageous in terms of software purchasing power and knowledge leverage.

The purpose for joining a consortium and following the CASMAC path was that the organisation had been aware since 1989 that it needed to replace its Student Records System (SRS). The organisation and the SRS were suffering from the constant need for reactive patching and fixes brought about by changing government policies and rapid organisational growth and change. However, the CASMAC consortium dissolved and the initiative was abandoned by the organisation in 1997. While work began on an upgrade to the SRS to meet Y2K requirements, this was regarded as an interim solution. As IS Professional A noted:

[The SRS] was on its last legs literally ...It couldn't keep up with the changing requirements of government easily ... It couldn't keep up with the requirements of Student 
Admin or the faculties ... the backlog of work was huge. The [organisation] needed to go more for [a] self-service model ... and they just couldn't do that.

The issue of replacing the SRS was raised at a meeting of senior executives in the organisation and a working party was formed. There was strong support from the Senior VP for integration of the organisation's administrative systems and the reengineering and development of better administrative procedures and practices. A decision was made to replace the SRS. Furthermore, as the IS Executive noted:

They wanted a student system and they wanted a finance system that was easy to integrate with the student system and while we're at it, we also need a HR system ... The top priority was still the student system.

In late 1998, the organisation issued a Request For Proposal (RFP) for the delivery of an administrative information system. Three vendors responded and performed software demonstrations onsite. An evaluation committee was formed to perform a high level functional analysis of the alternatives. In March 1999, the organisation approved the purchase of the ERP B1 Finance, Payroll and Student systems from Vendor B1 at a cost of approximately one and a half million dollars. The organisation also commissioned a detailed five week consultancy with the implementation partner, Consulting B1, to prepare an Implementation Planning Study (IPS). The purpose of this study was to identify project priorities, develop the business case in respect to benefits and costs and to scope all deliverables. As a result of this study, the eventual budget for the implementation of ERP B1 was established: this was approximately fourteen and a half million dollars. However, the implementation was subject to budget and time slippages and final costs to the organisation are estimated as high as twenty million dollars. Functionally, only the Student and most of the Finance systems were ever delivered, with extensive work required after the implementation (in excess of $\$ 1 \mathrm{M}$ ) to address issues with the Finance System.

\section{Evidence of information bias}

Two aspects of pre-decisional bias were evident. The first related to a strong bias against the option of building a new administrative system in-house. This was supported by comments from Academics A \& B, with Academic A stating that:

[The IS Executive] certainly presented the view forcefully that we shouldn't be building systems in-house, we should be buying them off the shelf because that was going to be a lot cheaper. My memory of the sorts of figures that were being talked about at this point in time were maybe a million bucks, maybe not even that much ...

I think what we had in our heads was that there were about three people in [the IS division] working on a student records system and maybe the calculations went like this: We've got three people over there working on this, that's 150 grand a year. We can buy one more cheaply because that's what we've been told therefore we would be spending about one hundred thousand dollars to get a new student records system.

This is consistent with the views of Academic B, who as chair of the evaluation committee, felt that his options were restricted. He stated that "I felt my hands were tied behind my back when for a start when I don't think I had the choice of looking at the fourth option". The "fourth option" he refers to was the option to build a new SRS in-house. He believes that by not examining this option, the decision process was restricted. He went on to add that "I felt that at that meeting it was almost cut-and-dry that it wouldn't be the idea of upgrading 'in-house'... I think [upgrading] the existing system wasn't considered".

The second aspect of pre-decisional bias was the strong bias towards ERP B1. The system had been demonstrated to at least one member of the senior executive who had then gone on to lobby 
other decision makers by distributing demonstration disks. IS Professional A, as a member of the evaluation committee, felt she had little impact on the decision she felt the decision had been made very early in the process and that everything that came after it was justification. She stated that "they had a team of more senior people that made the decisions ... you got the impression that they already had the decision they wanted ... and what we were doing was to justify that decision".

This is consistent with Academic A's statement that the IS Executive had handed him a disk with a demonstration of ERP A even before the RFP was called. There is another indication of this pre-decisional bias in IS Professional B's belief that he had little impact on the decision and the comment that "I think [the IS executive] was pretty keen to go with [ERP A] before we started the whole exercise". When asked how this was indicated, IS Professional B responded that "he was mentioning it a lot and that we need to go with ERPs because they are state-of-the-art and we need to introduce workflow procedures". He went on to add that "there must have been something operating on the decision process [and that the] decision to go with [ERP A] was made outside the group [of senior executives]". His belief was that the IS Executive had decided long before the evaluation process that ERP A would be selected and implemented.

\section{Cognitive bias at work}

Decision making surrounding the selection of the ERP was affected by a number of cognitive biases including reasoning by analogy, single outcome calculation and the criteria selection game. The interaction between these biases was related to issues surrounding risk mitigation and the reduction of uncertainty.

The decision making process had evidence of reasoning by analogy bias. Many of the decision makers had a poor understanding of the complexity and scale of the decision, least of all the potential organisation impacts and costs. The decision makers treated the decision like similar smaller decisions that they had made before. Academic B explained that:

I think they thought it was just like buying 'Word' and it works and this was a very different kettle of fish. I think that very few of those who made the decision had had exposure to the idea of buying a package before, let alone an ERP.

This form of reasoning by analogy also led to aspects of the illusion of control bias. In the face of uncertainty with decision makers having little experience in they type of decision they were making, they reduced uncertainty by reassuring themselves that they had made successful similar decisions in the past, in the same style. When asked how this had come about, IS Professional A stated that:

We had had pretty good success with the finance and [HR] systems and the users were happy with the functionality that we got from those systems ... Everyone was quite happy to go out and look for a product and evaluate what was out in the marketplace.

In effect, the decision makers believed that their previous successes in evaluating and implementing much smaller systems empowered them to make a good decision surrounding a much more complex product.

However, it is important to bear in mind that this bias was only reaffirming the pre-decisional biases and the preferred outcome. In order to justify the preferred outcome, single outcome calculation occurred. First of all, options were restricted, as IS Professional A explained:

Our philosophy was that we need to go and get something off the shelf because everybody needs the same things in a student system and we as an organisation would find it difficult to keep up with ... the government regulations. 
Secondly, there was a need to eliminate or restrict the choices so that there was really only one viable option. Apart from the preferred vendor, two other competing vendors were approached to provide a RFP whose products were, at face value, immature. However, a third vendor with a well known international product, although with limited local presence, was not invited to submit an RFP. Given the preferred vendor had only recently started to localise their SRS product, this seemed to be unusual.

Thirdly, information gathering was limited. This appears to have simplified the process that reduced the level of uncertainty. However, this had negative impacts on the evaluation and decision making process. IS Professional B maintained that the decision process was illogical and based on little substance, remarking that "I was a bit amazed that we went from the ... glossy brochure to putting in an order basically".

The final bias at work was the criteria selection game. In order to reduce uncertainty and to justify the preferred option, quantitative potential cost savings were used. It was reported that up to three hundred positions would be saved by implementing the ERP, primarily in casual and seasonal staff. This was quantified by the finance manager at approximately three million dollars per annum. However, the validity of this data is disputed and has not been substantiated postimplementation. IS Professional B was scathing about the estimates stating that "the decision to implement the ERP was based on cost savings which were invented ... I know the savings were absolutely fudged".

\section{Risk bias at work}

Much of the decision making process was concerned with avoiding or minimising risk to both the decision makers and the organisation. One problem evident with the decision process was that the people who evaluated the RFPs and products felt little ownership of the decision. The role of the evaluation team was to make a recommendation, however, as discussed previously, many already knew what the preferred option was be and felt it was their job to justify it. Moreover, information concerning some of the risks associated with the preferred product was either not communicated or was ignored by the senior executive making the final decision.

Part of this lack of risk awareness came about by the three layered decision making process. Academic $\mathrm{B}$ described the following steps in the decision process:

It went from [the evaluation committee] back up to the [Senior VP] who by that stage had formed another committee to keep looking at it. We knew that [Higher Education Organisation B1] was the first cab off the rank so we actually had an ISL (video link-up) with a number of key people at [Higher Education Organisation B1]

As the purchase price of the ERP was above the level the Senior VP could approve, there was a special presentation to University Council. They saw part of the [Higher Education Organisation B1] video plus presentations from various members of the executive review committee. They were also given an "executive summary of the [evaluation committee] report". The IS Executive confirms this version of events:

We then got the senior executive and some council members. We had them in a video conference link to [Higher Education Organisation B1] who had chosen [ERP B1]... We had the senior sponsor of the [Higher Education Organisation B1] project, maybe the vice-chancellor of [Higher Education Organisation B1] on the video conference link assuring the senior executives at this [organisation] that it was the right decision to make and why they'd made their decision, so, the [Senior Organisational Executive], then signed off because it was millions of dollars and exceeded the signing authority of the Senior VP so that's how we got ERP B1. 
Risk was mitigated by the executive decision makers talking to, and being reassured by, other executives at an organisation which was implementing the preferred product. However, information concerning implementation difficulties and increasing budget and project timelines from the same site were not communicated to the University Council.

A second risk bias that occurred could be best described as "follow the leader". Members of the evaluation committee were aware of the vendors who had a presence in Australia. Furthermore, they were aware that as a relatively small institution they needed to choose a vendor that would have a continued presence and one that, as part of a larger syndicate, they could lobby for product changes. Academic B explained by saying that:

We also looked at who within Australian [higher education institutions] has what packages and who is proposing to move to what packages. It was clear that [Vendor A] had the vast majority of the sites, for example they had [numerous institutions listed].

Vendor B1 had a significant presence in Australian higher education institutions at the time of the selection decision. The perceived risk in a "small" institution going with one of the smaller ERP vendors was explained by IS Professional:

We are too little to make a decision of a software vendor that no one else has picked. Part of the reason to buy [ERP B1] was that it was a financially viable organisation, it had an existing customer base in America and Australia with [a] Student [records module].... We had to go with what the other major [higher education organisations] were doing because that ... added to the decision making process but it also adds to the clout when we need changes to the software ... we've got a bigger user group ... We couldn't gamble on going on a smaller provider.

\section{Uncertainty bias at work}

Given the complexity of the ERP selection decision, it would be easy to assume that there would be a high degree of uncertainty thus visible effects of information overload. However, in this instance, quite the contrary occurred. It appears that this organisation self-censored the level of information available to decisions maker in order to decrease uncertainty. This had negative consequences on the evaluation process. IS Professional B believed that the cursory review completed by the evaluation committee had limitations, stating that "as far as I was concerned the advisory committee was only advising on what they thought was the best solution ... they didn't look into it in terms of suitability in detail".

He went on to add that "we didn't actually talk to anybody who was using the product on the sites or anything like ... it was really the glossy brochure without even the test drive". Academic B also admitted that the evaluation was not necessarily as rigorous as it could have been. Much of the bias at work in the ERP selection decision can be traced to the high degree of underlying uncertainty and the efforts to, at face value, minimise this uncertainty.

\section{Effects of bias in Organisation B}

This case challenges some conventional understandings about how a complex IS decision such as the selection of an ERP would be performed. A boundedly rational approach to the decision making process would see decision makers gather as much information as they thought they needed to inform the decision. However, in this instance, little information was gathered. Interviewees admit that they operated on sales information and demonstrations. On this basis, they selected a product. Inexplicably, this almost appears to be an instance of "information underload" although conforms with what theoretically happens in the face of uncertainty (Daft \& Lengel, 1990). Interviewees gave examples of where information was excluded or options were restricted from the 
decision process, for example, not considering either the other "large" ERP in the market or the build in-house option. These are examples of pre-decisional bias and single outcome calculation.

Consistent with the literature was the need to make the decision processes appear rational. The appearance of rationality can often be achieved by using hard, tangible justifications for a decision. One such "hard" justification given in this case was cost savings: this is consistent with the criteria selection game. However, it is evident form the statements of interviewees there were a number of soft factors in the form of the biases already described. Perhaps more telling is that it is doubtful if any of the cost savings quoted were ever achieved.

From the evidence of strong pre-decisional bias, in combination with other cognitive biases and the effects of risk and uncertainty, there appears to have never been a question of which product would have been selected. Many in the organisation regard the decision outcomes as poor, and the projected efficiencies are yet to be realised. It is clear that bias in this decision making process restricted the options and created negative information filters.

\section{Discussion}

The two cases differ in many ways; however, the basic decision was the same. Both organisations were faced with the selection of a major piece of IS infrastructure that would have considerable organisation impacts. Both organisations had the personnel and resources to effectively evaluate systems. Both organisations faced similar time constraints. This raises the question: why would bias help a decision making process in one organisation and hinder it in another?

The research findings indicate that the way in which bias affects decisions outcomes is related to a number of contextual influences. These influences include organisational culture, organisational history, organisational relationships, internal organisational structures, politics, composition of the decision making body and organisational size. In keeping with the model depicted in Figure 1, contextual factors play a role in the way bias affects information and the way bias affects the way decisions occur. The research findings indicate that the same bias elicits different results, depending on the context in which it is applied. An over-arching theme to the findings is that uncertainty biases have a considerable role in determining how other bias groups are applied.

\section{Information Bias}

In Organisation A, the organisational history and level of trust developed between the organisation and the existing vendor were strong contextual influences in the decision making and led to pre-decisional bias. This formation of a preference before a formal evaluation occurs is not unusual (Russo et al., 1998). In this instance, while the existing vendor relationship contextually influenced the decision, it also biased information. Information from the vendor was regarded as reliable and exhibited lower levels of uncertainty than that from other vendors. A pre-decisional bias in the form of "bench-marking" occurred whereby an implicit comparison of information from other vendors was performed against the existing vendors, even before it was formally evaluated. From this, it could be argued that this bias was justified and produce positive effects by reducing uncertainty and simplifying the decision process.

However, in Organisation B, the pre-decisional bias was based on a product awareness that resulted from political lobbying within the organisation. A general perception had been developed in the minds of senior decision makers that other organisations were using the product successfully and that they had not encountered difficulties or cost over-runs during implementation. Another element of Organisation B's pre-decisional bias was the automatic exclusion of the in-house build option. This was based on the contextual elements of organisational history and culture whereby the internal IS department was viewed by some senior managers as incompetent. This bias not only eliminated the option of building in-house with existing staff, but it also removed 
several options associated with sub-contracting out the requirement of the organisation. It also limited the amount of input that internal stakeholders may have had on the assessment and evaluation.

The overall complexity of the decision caused a great degree of uncertainty in Organisation B and this distorted the information that fed the informing system. The extensive use of cognitive games to simply information and make the decision comparable with previous decisions created an artificial decision environment. The use of a poorly informed decision making body, a contextual factor, and the reliance of selective, high-level information demonstrates the presence of cognitive and risk biases. It is particularly interesting that in order to achieve cognitive fit and reduce uncertainty (Mintzberg, 1972; Umanath \& Vessey, 1994) senior decision makers justified their decisions by nominating the views of their peers at other organisations and estimates of financial savings.

\section{Process Bias}

Another consideration when evaluating the effects of bias is to determine if there was scope for them to impact on the decision process in any meaningful way. As previously discussed, the findings indicated that bias affected the decision processes. The extent to which bias affected the process was dependent on organisational structures and culture and these structures dictated how rigid and transparent the decision process was.

Although staff in both organisations stated that they had formal evaluation processes, documentation and supporting interviews indicated that Organisation A had a more rigid and transparent process than Organisation B. This included formally recorded score cards and a weighting system. However, while organisation $\mathrm{B}$ had a report from the evaluation committee, the actual process of evaluation and the data used to justify the recommendation was not formally recorded. This was compounded by the loose transition from a recommendation from the evaluation committee to the senior executive to the verbal presentation to University Council. At every phase in this process, human bias could be, and was, introduced. The extent to which this process was flawed was highlighted by criticism in the organisation's annual external audit.

Organisation A had an existing culture of thorough evaluation practices with the organisational structures in place to undertake them. Organisation B had a more informal culture and had disjointed organisational structures in place that permitted a looser evaluation. However, why were structures not put in place in Organisation B to facilitate a more rigid and transparent process? Data indicates that this was not necessarily in the best interest of some of the key decision makers, especially those with strong pre-decisional bias. To reduce the levels of uncertainty surrounding the decision, the evaluation process was devolved and information was selectively filtered up to final key decision makers. The overall complexity of the decision, the uncertainty surrounding it in and the combination with the lack of structure and ownership culture allowed this to happen.

\section{Development of the model}

In order to capture the interaction between contextual factors and bias, a refinement of the decision making model depicted in Figure 1 is suggested. The revised model, depicted in Figure 2, takes into account contextual factors such as organisational history that can mitigate or alter the effects of the bias groups. 


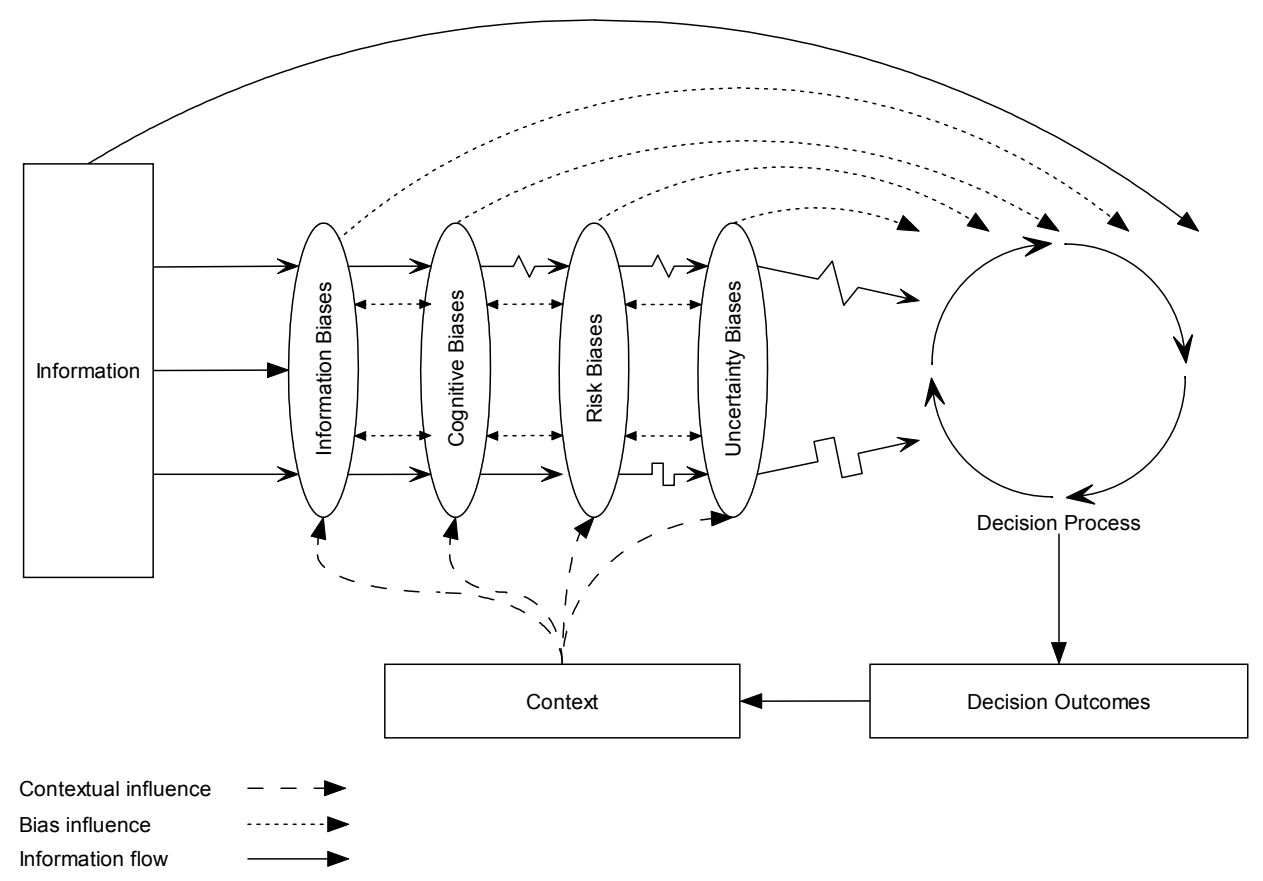

Figure 2 - Bias in an informing system (contextual model)

In the model, decision outcomes also feed into context as they become part of the organisational history and affect other contextual factors such as politics and organisational structures. The revised model also proposes an interaction between bias groups. Although in this study only uncertainty biases were observed to perpetuate other biases, it seems likely that other bias groups interact with one another. It should be noted that the direct interaction between contextual factors and decision processes has not been captured in this model as it is beyond the scope of this study.

\section{Conclusions}

The results of this study clearly indicate that bias is a function of the contextual influences such as organisational culture, organisational history, organisational relationships, internal organisational structures, politics, composition of the decision making body and organisational size. The extent to which this bias will affect a decision will depend on how rigid and transparent the decision making process is or needs to be. It is also clear that biases can be linked to the level of uncertainty surrounding a decision. That is, when there is a degree of uncertainty, individuals will resort to using biases to deal with the uncertainty: at times this may increase organisational uncertainty but it reduces the apparent risk to the individuals. From these two studies, it is apparent that lower levels of uncertainty, as in the case of Organisation A, lead to a lower susceptibility to entertain bias. However, in organisations where there is a high degree of uncertainty surrounding the decision, bias has more potential to influence decision making. This potential influence may be tempered with more rigor and transparency in the evaluation and decision making process.

In summary, this paper has described the effects of bias in two organisations making similar IS selection decisions. It is clear from this study that reality and the data captured by decision makers can often be two very different things. We have determined that bias within an informing system may have a positive effect on a decision if the bias is based on reliable experiences or information. However, bias can also distort information gathering within an informing system and lead to sub-optimal outcomes. In order to temper the effects of bias on the way information is gathered 
and evaluated, rigorous and transparent IS evaluation practices must be used. However, for these practices to occur, contextual factors such as a supportive organisational culture and structure must be in place.

\section{Limitations}

The research from this paper is drawn from a larger study however for the purposes of reporting, much of the detail has been condensed. The sample of organisations and interviewees are indicative of the results obtained from other projects and since no attempt has been made to perform statistical analysis, the small sample size has no effect on a qualitative study of this type. The methodology relies on the honesty and openness of the participants of the study however the analysis technique has been designed to triangulate facts through multiple verifications. However, it is recognised that in this type of qualitative study, the authors' own biases become part of the interpretive process. In doing so, every attempt has been made to present the findings as part of an explanative narrative, and as Klein \& Myers (1999) note, prejudices and biases may in themselves lead to a better understanding of research findings.

\section{References}

Bainbridge, S. M. (2002). Why a board? Group decision making in corporate governance. Vanderbilt Law Review, 55(1), 1-54.

Ballantine, J. A., \& Stray, S. (1999). Information systems and other capital investments: Evaluation practices compared. Leadership and Organization Development Journal, 12(1/2), 78-93.

Bannister, F., \& Remenyi, D. (1999). Value perception in IT investment decisions. The Electronic Journal of Information Systems Evaluation, 2(2).

Brindle, M. (1999). Games decision makers play. Management Decision, 37(8), 604-612.

Buchanan, J., \& Kock, N. (2000). Information overload: A decision making perspective. Paper presented at the Multiple Criteria Decision Making 2000, Ankara.

Chan, S. Y. (2002). The effect of information load and presentation format on managerial decision quality. Hong Kong: Hong Kong Baptist University.

Daft, R. L., \& Lengel, R. H. (1990). Information richness: A new approach to managerial behavior and organization design. In L. L. Cummings \& B. M. Straw (Eds.), Information and cognition in organisations (pp. 243-285). London, England: JAI Press Inc.

Doherty, N., \& King, M. (2001). The treatment of organisational issues in systems development projects: The implications for the evaluation of information technology investments. The Electronic Journal of Information Systems Evaluation, 4(1).

Duhaime, I. M., \& Schwenk, C. R. (1985). Conjectures on cognitive simplification in acquisition and divestment decision making. Academy of Management. The Academy of Management Review (pre1986), 10(2), 287-295.

Eagly, A. H., \& Chaiken, S. (1993). The psychology of attitudes. Orlando: Harcourt Brace College Publishers.

Falzon, L., Zhang, L., \& Davies, M. (2000, 24-26 October). A policy analysis approach to operational level course of action analysis. Paper presented at The 5th International Command and Control Technology Symposium, Canberra, Australia.

Grise, M.-L., \& Gallupe, R. B. (2000). Information overload: Addressing the productivity paradox in faceto-face electronic meetings. Journal of Management Information Systems, 16(3), 157-186.

Irani, Z., \& Love, P. E. D. (2001). The propagation of technology management taxonomies for evaluating investments in information systems. Journal of Management Information Systems, 17(3), 161-177. 
Iselin, E. R. (1993). The effects of the information and data properties of financial ratios and statements on managerial decision quality. Journal of Business Finance and Accounting, 20(2), 249-266.

Jacoby, J. (1977). Information load and decision quality: Some contested issues. Journal of Marketing Research, 14(4), 569-573.

Jamieson, K., \& Hyland, P. (2004, December 1-3 2004). IS decisions: Identifying information sources, considerations and justifications. Paper presented at The 15th Australasian Conference on Information Systems, Hobart, Tasmania Australia.

Klein, H. K., \& Myers, M. D. (1999). A set of principles for conducting and evaluating interpretive field studies in information systems. MIS Quarterly, 23(1), 67-93.

Lin, C., \& Pervan, G. (2001). IS/IT investment evaluation and benefits realisation issues in government organisation. Paper presented at The Twelfth Australasian Conference on Information Systems, Coffs Harbour, NSW Australia.

Mahaney, R. C., \& Lederer, A. L. (1999). Runaway information systems projects and escalating commitment. Paper presented at the Special Interest Group on Computer Personnel Research Annual Conference, New Orleans, Lousiana, USA.

March, J. G., \& Shapira, Z. (1987). Managerial perspectives on risk and risk taking. Management Science, 33(11), 1404-1418.

March, J. G., \& Simon, H. A. (1958). Organizations. New York: John Wiley \& Sons.

Maritan, C. (2001). Capital investment as investing in organizational capabilities: An empirically grounded process model. Academy of Management Journal, 44(3), 513-532.

McCormick, E. (1970). Human Factors Engineering. New York: McGraw-Hill.

Mintzberg, H. (1972). The manager's job: Folklore and fact. In J. J. Gabarro (Ed.), Managing people and organisations (pp. 13-32). Boston: McGraw-Hill.

Mintzberg, H., Waters, J., Pettigrew, A. M., \& Butler, R. (1990). Studying deciding: An exchange of views between Mintzberg and Waters, Pettigrew, and Butler. Organization Studies, 11(1), 1-15.

Myers, M. D. (1994a). Dialectical hermeneutics: A theoretical framework for the implementation of information systems. Information Systems Journal, 5, 51-70.

Myers, M. D. (1994b). A disaster for everyone to see: An interpretive analysis of a failed IS project. Accounting, Management and Information Technology, 4(4), 185-201.

O'Reilly, C. A. I. (1990). The use of information in organizational decision making. In L. L. Cummings \& B. M. Straw (Eds.), Information and cognition in organizations (pp. 89-125). London: JAI Press Ltd.

Russo, J. E., Meloy, M. G., \& Medvec, V. H. (1998). Predecisional distortion of product information. JMR, Journal of Marketing Research, 35(4), 438-451.

Schroder, H. M., Driver, M. J., \& Streufert, S. (1967). Human information processing. New York: Holt, Rinehart \& Winston.

Simon, H. A. (1967). A behavioral model of rational choice. In M. Alexis \& C. Z. Wilson (Eds.), Organizational Decision Making (pp. 174-184). New Jersey: Prentice-Hall.

Simon, H. A. (1987). Making management decisions: The role of intuition and emotion. The Academy of Management Executive, 1(1), 57-63.

Simon, H. A. (1997). Administrative behaviour: A study of decision-making processes in administrative organizations (4th ed.). New York: The Free Press.

Simons, R. H., \& Thompson, B. M. (1998). Strategic determinants: The context of managerial decision making. Journal of Managerial Psychology, 13(1, 2), 7-21.

Smith, H. J., \& Keil, M. (2003). The reluctance to report bad news on troubled software projects: A theoretical model. Information Systems Journal, 13, 69-95. 
Swain, M. R., \& Haka, S. F. (2000). Effects of information load on capital budgeting decisions. Behavioral Research in Accounting, 12, 171-199.

Umanath, N. S., \& Vessey, I. (1994). Multiattribute data presentation and human judgment: A cognitive fit perspective. Decision Sciences, 25(5/6), 795-823.

Verville, J., \& Halingten, A. (2002). An investigation of the decision process for selecting an ERP software: The case of ESC. Management Decision, 40(3), 206-216.

Vessey, I. (1994). The effect of information presentation on decision making: A cost-benefit analysis. Information and Management, 27, 103-119.

Yin, R. K. (1994). Case Study Research: Design and Methods (2nd ed.). Newbury Park, California: Sage Publications.

\section{Biographies}

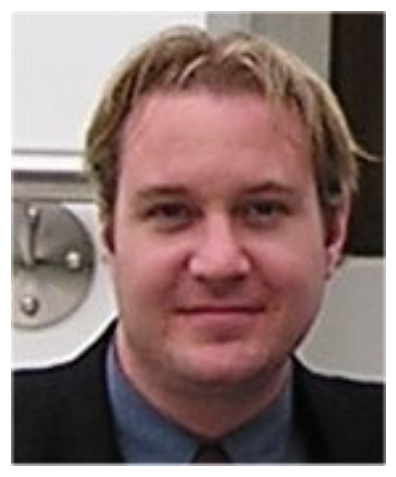

Kieren Jamieson is a Computer Science lecturer and IS Management $\mathrm{Ph} . \mathrm{D}$ candidate at Central Queensland University. His research focuses on how complex IS decisions occur and the factors that affect the decision makers. His research to date has directly resulted in the publication of four refereed research papers and one book chapter.

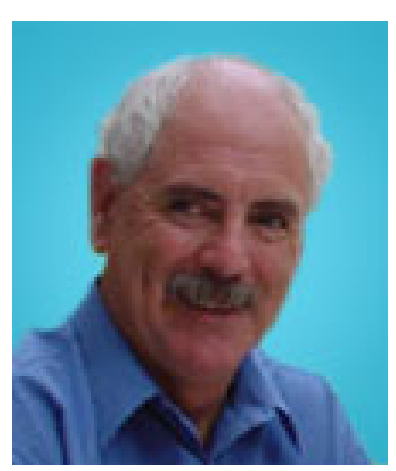

Dr Paul Hyland is Professor of Management in the Faculty of Business and Informatics at Central Queensland University. Dr Hyland is active and a Board member in CINet (the Continuous Innovation Network), a global network set up to bring together researchers and industrialists working in the area of continuous innovation. CINet disseminates a new way of thinking about management and the organisation of work. He is currently the international coordinator of a global project surveying continuous improvement activities in Europe, Australia and SE Asia. He has published over 40 refereed journal articles, four book chapters and over 100 international conference papers. 